\title{
NESTED DERIVATIVES: A SIMPLE METHOD FOR COMPUTING SERIES EXPANSIONS OF INVERSE FUNCTIONS
}

\author{
DIEGO DOMINICI
}

Received 28 March 2003

\begin{abstract}
We give an algorithm to compute the series expansion for the inverse of a given function. The algorithm is extremely easy to implement and gives the first $N$ terms of the series. We show several examples of its application in calculating the inverses of some special functions.
\end{abstract}

2000 Mathematics Subject Classification: 40-04, 33F10, 41A58.

1. Introduction. The existence of series expansions for inverses of analytic functions is a well-known result of complex analysis [17]. The standard inverse function theorem, a proof of which can be found, for example, in [12], is as follows.

THEOREM 1.1. Let $h(x)$ be analytic for $\left|x-x_{0}\right|<R$, where $h^{\prime}\left(x_{0}\right) \neq 0$. Then $z=h(x)$ has an analytic inverse $x=H(z)$ in some $\varepsilon$-neighborhood of $z_{0}=$ $h\left(x_{0}\right)$.

In the case when $x_{0}=z_{0}=0,|h(x)| \leq M$ for $|x|<R$, and $h^{\prime}(0)=a$, Redheffer [25] has shown that it is enough to take $\varepsilon=(1 / 4)\left((a R)^{2} / M\right)$.

However, the procedure to obtain the actual series is usually very difficult to implement in practice. Under the conditions of Theorem 1.1, the two standard methods to compute the coefficients $b_{n}$ of

$$
h^{-1}(z)=H(z)=\sum_{n \geq 0} b_{n}\left(z-z_{0}\right)^{n}
$$

are reversion of series [16, 26, 33] and Lagrange's theorem. The first one requires expanding $h(x)$ around $x_{0}$

$$
h(x)=\sum_{n \geq 0} a_{n}\left(x-x_{0}\right)^{n}
$$

and then solving for $b_{n}$ in the equation

$$
z=\sum_{n \geq 0} a_{n}\left[\sum_{n \geq 0} b_{n}\left(z-z_{0}\right)^{n}-x_{0}\right]^{n}
$$


by equating powers of $z$ and taking into account that $a_{0}=z_{0}$ and $b_{0}=x_{0}$. This method is especially useful if all what is known about $h(x)$ are the first few $a_{n}$. When $x_{0}=z_{0}=0$ and $a_{1}=a$, it was shown by Whittaker [34] that

$$
\begin{gathered}
b_{1}=\frac{1}{a}, \quad b_{2}=-\frac{a_{2}}{a^{3}}, \quad b_{3}=\frac{1}{3 ! a^{5}}\left|\begin{array}{ccc}
3 a_{2} & a \\
6 a_{3} & 4 a_{2}
\end{array}\right|, \ldots, \\
b_{n}=\frac{(-1)^{n-1}}{n ! a^{2 n-1}}\left|\begin{array}{ccccc}
n a_{2} & a & 0 & 0 & \ldots \\
2 n a_{3} & (n+1) a_{2} & 2 a & 0 & \ldots \\
3 n a_{4} & (2 n+1) a_{3} & (n+2) a_{2} & 3 a & \ldots \\
4 n a_{5} & (3 n+1) a_{4} & 2(n+1) a_{3} & (n+3) a_{2} & \ldots \\
\vdots & \vdots & \vdots & \vdots & \ddots
\end{array}\right|,
\end{gathered}
$$

where $|\cdot| \equiv \operatorname{det}(\cdot)$.

In Example 4.7, we show how to get the $b_{n}$ in terms of the $a_{n}$ using our method.

A computer system like Maple can reverse the power series of $h(x)$, provided $h(x)$ is not too complicated, by using the command

$$
\begin{aligned}
& >\text { Order }:=N+1 ; \\
& >\operatorname{solve}\left(\operatorname{series}\left(h(x), x=x_{0}, N+1\right)=z, x\right)
\end{aligned}
$$

where $N$ is the number of terms wanted. Fast algorithms of order $(n \log n)^{3 / 2}$ for reversion of series have been analyzed by Brent and Kung [5, 6]. The multivariate case has been studied by several authors [4, 8, 14, 21], and Wright [35] has studied the connection between reversion of power series and "rooted trees."

The second and more direct method is Lagrange's inversion formula [1]

$$
b_{n}=\left.\frac{1}{n !} \frac{d^{n-1}}{d x^{n-1}}\left\{\left[\frac{x-x_{0}}{h(x)-z_{0}}\right]^{n}\right\}\right|_{x=x_{0}} .
$$

Unfortunately, more direct does not necessarily mean easier, and except for some simple cases Lagrange's formula (1.6) is extremely complicated for practical applications. The $q$-analog (a mathematical expression parameterized by $q$ which generalizes an expression and reduces to it in the limit $q \rightarrow 1^{+}$) of (1.6) has been studied by various authors $[2,18,19,20]$ and a unified approach to both the regular and $q$-analog formulas has been obtained by Krattenthaler [23]. There has also been a great deal of attention to the asymptotic expansion of inverses [27, 28, 31, 32].

In this note, we present a simple, easy to implement method for computing the series expansion for the inverse of any function satisfying the conditions of Theorem 1.1, although the method is especially powerful when $h(x)$ has 
the form

$$
h(x)=\int_{a}^{x} g(x) d x
$$

and $g(x)$ is some function simpler than $h(x)$. Since this is the case for many special functions, we will present several such examples. This note is organized as follows.

In Section 2, we define a sequence of functions $\mathbb{T}^{n}[f](x)$, obtained from a given one $f(x)$, that we call "nested derivatives," for reasons which will be clear from the definition. We give a computer code for generating the nested derivatives and examples of how $\mathfrak{D}^{n}[f](x)$ look for some elementary functions. Section 3 shows how to compute the nested derivatives by using generating functions. We present some examples and compare the results with those obtained in Section 1.

Section 4 contains our main result of the use of nested derivatives to compute power series of inverses. We test our result with some known results and we apply the method for obtaining expansions for the inverse of the error function, the incomplete gamma function, the sine integral, and other special functions.

\section{Definitions}

Definition 2.1. The $n$th nested derivative $\mathbb{C}^{n}[f](x)$ of the function $f(x)$ is defined by the following recursion:

$$
\begin{gathered}
\mathbb{D}^{0}[f](x) \equiv 1, \\
\mathbb{D}^{n}[f](x)=\frac{d}{d x}\left[f(x) \times \mathbb{D}^{n-1}[f](x)\right], \quad n \geq 1 .
\end{gathered}
$$

Proposition 2.2. The nested derivative $\mathbb{1}^{n}[f](x)$ satisfies the following basic properties.

(1) For $n \geq 1$, 过 ${ }^{n}[\kappa] \equiv 0$, with $\kappa$ constant.

(2) For $n \geq 0$, 书 ${ }^{n}[\kappa f](x)=\kappa^{n} \mathbb{1}^{n}[f](x)$, with $\kappa$ constant.

(3) For $n \geq 1$, $\mathbb{D}^{n}[f](x)$ has the following integral representation:

$$
\mathfrak{D}^{n}[f](x)=\frac{1}{(2 \pi i)^{n}} \oint_{C_{1}} \oint_{C_{2}} \cdots \oint_{C_{n}} \frac{f\left(z_{n}\right)}{\left(z_{n}-x\right)^{2}} \prod_{k=1}^{n-1} \frac{f\left(z_{k}\right)}{\left(z_{k}-z_{k+1}\right)^{2}} d z_{n} \cdots d z_{1} \text {, }
$$

where $C_{k}$ is a small loop around $x$ in the complex plane.

Proof. Properties (1) and (2) follow immediately from the definition of $\mathbb{1}^{n}[f](x)$. 
To prove (3) we use induction on $n$. For $n=1$, the result follows from Cauchy's formula

$$
\mathbb{D}^{1}[f](x)=\frac{d f}{d x}=\frac{1}{2 \pi i} \oint_{C_{1}} \frac{f\left(z_{1}\right)}{\left(z_{1}-x\right)^{2}} d z_{1} \text {. }
$$

Assuming that the result is true for $n$ and using (2.2),

$$
\begin{aligned}
\mathbb{D}^{n+1}[f](x) & =\frac{d}{d x}\left[f(x) \times \mathbb{D}^{n}[f](x)\right] \\
& =\frac{1}{2 \pi i} \oint_{C_{n+1}} \frac{f\left(z_{n+1}\right) \mathbb{C}^{n}[f]\left(z_{n+1}\right)}{\left(z_{n+1}-x\right)^{2}} d z_{n+1} \\
& =\frac{1}{(2 \pi i)^{n+1}} \oint_{C_{1}} \cdots \oint_{C_{n+1}} \frac{f\left(z_{n+1}\right)}{\left(z_{n+1}-x\right)^{2}} \frac{f\left(z_{n}\right)}{\left(z_{n}-z_{n+1}\right)^{2}} \\
& =\frac{1}{(2 \pi i)^{n+1}} \oint_{C_{1}} \cdots \oint_{C_{n+1}} \frac{f\left(z_{n+1}\right)}{\left(z_{n+1}-x\right)^{2}} \\
& \times \prod_{k=1}^{n-1} \frac{f\left(z_{k}\right)}{\left(z_{k}-z_{k+1}\right)^{2}} d z_{n+1} \cdots d z_{1} \\
&
\end{aligned}
$$

Algorithm 2.3 (the $\mathbb{D}$ algorithm). The following Maple procedure implements the recurrence relation (2.2). We define $d(k)=\mathbb{D}^{k}[f](x)$, where $N$ is the number of terms desired:

$$
\begin{aligned}
& >d(0):=1 \\
& >\text { for } k \text { from } 0 \text { to } N \text { do: } \\
& >d(k+1):=\operatorname{simplify}(\operatorname{diff}(f * d(k), x)): \\
& >\operatorname{print}(k+1, d(k+1)): \\
& >\text { od: }
\end{aligned}
$$

EXAMPLE 2.4. Let the function $f(x)=x$, then

$$
\begin{gathered}
\mathbb{D}^{1}[f](x)=1, \\
\mathfrak{D}^{2}[f](x)=1, \\
\vdots \\
\mathbb{D}^{n}[f](x)=1 .
\end{gathered}
$$


EXAMPLE 2.5. Let the power function $f(x)=x^{r}, r \neq 1$, then

$$
\begin{aligned}
\mathbb{D}^{1}[f](x) & =r x^{r-1}, \\
\mathbb{D}^{2}[f](x) & =r(2 r-1) x^{2(r-1)}, \\
v \mathbb{D}^{3}[f](x) & =r(2 r-1)(3 r-2) x^{3(r-1)}, \\
& \vdots \\
\mathbb{D}^{n}[f](x) & =\prod_{j=1}^{n}[j r-(j-1)] x^{n(r-1)} \\
& =(r-1)^{n} \frac{\Gamma(n+1+1 /(r-1))}{\Gamma(1+1 /(r-1))} x^{n(r-1)} .
\end{aligned}
$$

Notice that when $r=k /(k+1), k=1,2, \ldots$, the sequence of nested derivatives has only $k+1$ nonzero terms.

$$
\mathbb{L}^{n}[f](x)= \begin{cases}\frac{k !}{(k-n) !(k+1)^{n}} x^{-(n /(k+1))}, & 1 \leq n \leq k, \\ 0, & n \geq k+1 .\end{cases}
$$

EXAMPLE 2.6. Let the exponential function $f(x)=e^{r x}$, then

$$
\begin{aligned}
\mathbb{D}^{1}[f](x) & =r e^{r x}, \\
\mathbb{D}^{2}[f](x) & =2 r^{2} e^{2 r x}, \\
\mathbb{D}^{3}[f](x) & =6 r^{3} e^{3 r x}, \\
\vdots & \\
\mathbb{D}^{n}[f](x) & =n ! r^{n} e^{n r x} .
\end{aligned}
$$

3. Generating functions. Generating functions provide a valuable method for computing sequences of functions defined by an iterative process, we will use them to calculate $\mathbb{1}^{n}[f](x)$. In the sequel, we will implicitly assume that the generating function series converges in some small disc around $z=0$.

THEOREM 3.1. Given $h(x)=\int(1 / f(x)) d x$, its inverse $H(x)=h^{-1}(x)$, and the exponential generating function $G(x, z)=\sum_{n \geq 0} \mathbb{D}^{n}[f](x)\left(z^{n} / n !\right)$, it follows that

$$
G(x, z)=\frac{1}{f(x)}(f \circ H)[z+h(x)]
$$


Proof. Taking (2.2) into account gives

$$
\begin{aligned}
\frac{\partial}{\partial x}[f(x) G(x, z)] & =\sum_{n \geq 0} \frac{d}{d x}\left[f(x) \times \mathbb{D}^{n}[f](x)\right] \frac{z^{n}}{n !} \\
& =\sum_{n \geq 0} \mathbb{D}^{n+1}[f](x) \frac{z^{n}}{n !}=\sum_{n \geq 1} \mathbb{D}^{n}[f](x) \frac{z^{n-1}}{(n-1) !} \\
& =\frac{\partial}{\partial z} \sum_{n \geq 0} \mathbb{D}^{n}[f](x) \frac{z^{n}}{n !}=\frac{\partial}{\partial z} G(x, z) .
\end{aligned}
$$

Hence, the generating function satisfies the PDE

$$
\frac{\partial(f \times G)}{\partial x}=\frac{\partial G}{\partial z}
$$

with general solution

$$
G(x, z)=\frac{1}{f(x)} g[z+h(x)]
$$

where $g(z)$ is an arbitrary analytic function. Invoking the boundary condition $G(x, 0)=\mathbb{W}^{0}[f](x)=1$, (3.4) gives

$$
\frac{1}{f(x)} g[h(x)]=1,
$$

and therefore,

$$
f(x)=(g \circ h)(x) .
$$

If we take $x=H(w)$, then

$$
(f \circ H)(w)=(g \circ h \circ H)(w)=g(w)
$$

and the theorem follows.

EXAMPLE 3.2 (the function $f(x)=x)$. Here $h(x)=\int(1 / x) d x=\ln (x), H(x)=$ $e^{x}$, and from (3.1) it follows that

$$
G(x, z)=\frac{1}{x} \exp [z+\ln (x)]=e^{z} .
$$

We could obtain the same result from Example 2.4 by summing the series

$$
G(x, z)=\sum_{n \geq 0} 1 \frac{z^{n}}{n !}=e^{z}
$$

EXAMPLE 3.3 (the power function $f(x)=x^{r}, r \neq 1$ ). Now,

$$
h(x)=\int x^{-r} d x=\frac{x^{1-r}}{1-r}, \quad H(x)=[(1-r) x]^{1 /(1-r)},
$$


and we get

$$
\begin{aligned}
G(x, z) & =x^{-r}\left\{\left[(1-r)\left(z+\frac{x^{1-r}}{1-r}\right)\right]^{1 /(1-r)}\right\}^{r} \\
& =\left[\frac{(1-r) z+x^{1-r}}{x^{1-r}}\right]^{r /(1-r)} \\
& =\left[1+(1-r) x^{r-1} z\right]^{r /(1-r)} .
\end{aligned}
$$

Expanding in series around $z=0$, we recover the result from Example 2.5.

If $r /(1-r)=k$, that is, $r=k /(k+1), k=0,1, \ldots$, then $G(x, z)$ is a polynomial of degree $k$ in $z$, and hence,

$$
\mathbb{P}^{n}[f](x)=0, \quad n \geq k+1,
$$

as we have already observed in Example 2.5.

Given the particular form of the function $h(x)$ in Theorem 3.1, we can get alternative expressions for (3.1) which sometimes are easier to employ.

COROLlary 3.4. Let $h(x)=\int(1 / f(x)) d x$, its inverse $H(x)=h^{-1}(x)$, and the exponential generating function $G(x, z)=\sum_{n \geq 0} \mathbb{1}^{n}[f](x)\left(z^{n} / n !\right)$. Then,

(i) $G(x, z)=1 / f(x) H^{\prime}[z+h(x)]$,

(ii) $G(x, z)=d / d x H[z+h(x)]$.

Proof. (i) By definition, $(h \circ H)(x)=x$, so

$$
h^{\prime}[H(x)] H^{\prime}(x)=1
$$

but since $h(x)=\int(1 / f(t)) d t$, we get

$$
\frac{1}{f[H(x)]} H^{\prime}(x)=1
$$

or

$$
(f \circ H)(x)=H^{\prime}(x)
$$

and therefore,

$$
\begin{aligned}
G(x, z) & =\frac{1}{f(x)}(f \circ H)[z+h(x)] \\
& =\frac{1}{f(x)} H^{\prime}[z+h(x)] .
\end{aligned}
$$

(ii) Using the chain rule,

$$
\frac{d}{d x} H[z+h(x)]=H^{\prime}[z+h(x)] h^{\prime}(x)=H^{\prime}[z+h(x)] \frac{1}{f(x)}
$$

and the conclusion follows from part (i). 
4. Applications. We now state our main result.

THEOREM 4.1. Let $h(x)=\int_{a}^{x}(1 / f(t)) d t$, with $f(a) \neq 0, \pm \infty$, and its inverse $H(x)=h^{-1}(x)$. Then,

$$
H(z)=a+f(a) \sum_{n \geq 1} \mathbb{D}^{n-1}[f](a) \frac{z^{n}}{n !},
$$

where $|z|<\varepsilon$, for some $\varepsilon>0$.

Proof. We first observe that since $h(a)=0$, we have $H(0)=a$, and from Corollary 3.4(i),

$$
G(a, z)=\frac{1}{f(a)} H^{\prime}[z+h(a)]=\frac{1}{f(a)} H^{\prime}(z),
$$

where

$$
G(a, z)=\sum_{n \geq 0} \mathbb{D}^{n}[f](a) \frac{z^{n}}{n !}
$$

Hence,

$$
\begin{aligned}
H(z) & =H(0)+\int_{0}^{z} f(a) \sum_{n \geq 0} \mathbb{D}^{n}[f](a) \frac{t^{n}}{n !} d t \\
& =a+f(a) \sum_{n \geq 0} \mathbb{D}^{n}[f](a) \frac{z^{n+1}}{(n+1) !} \\
& =a+f(a) \sum_{n \geq 1} \mathbb{D}^{n-1}[f](a) \frac{z^{n}}{n !} .
\end{aligned}
$$

EXAMPLE 4.2 (the natural logarithm function). Let $f(x)=e^{-x}$ with $a=0$. We have $f(0)=1$,

$$
h(x)=\int_{0}^{x} e^{t} d t=e^{x}-1, \quad H(x)=\ln (x+1),
$$

and from Example 2.6,

$$
\mathbb{1}^{n}[f](0)=(-1)^{n} n ! .
$$

Hence, from (4.1), we get the familiar formula

$$
\ln (z+1)=\sum_{n \geq 1}(-1)^{n-1} \frac{z^{n}}{n} .
$$

EXAMPLE 4.3 (the tangent function). Let $f(x)=x^{2}+1$ with $a=0$. Now $f(0)=1$,

$$
h(x)=\int_{0}^{x} \frac{1}{t^{2}+1} d t=\arctan (x), \quad H(x)=\tan (x)
$$


and (4.1) implies that

$$
\tan (z)=\sum_{n \geq 1} \mathbb{P}^{n-1}\left[x^{2}+1\right](0) \frac{z^{n}}{n !} .
$$

Therefore,

$$
\begin{gathered}
\mathfrak{D}^{2 k+1}\left[x^{2}+1\right](0)=0, \quad k \geq 0, \\
\mathfrak{D}^{2 k}\left[x^{2}+1\right](0)=\frac{2}{k+1} 4^{k}\left(4^{k+1}-1\right)\left|B_{2(k+1)}\right|, \quad k \geq 1,
\end{gathered}
$$

where $B_{k}$ are the Bernoulli numbers [1].

REMARK 4.4. From Example 2.5, we recall that

$$
\mathbb{L}^{n}\left[x^{2}\right](x)=(n+1) ! x^{n},
$$

and consequently,

$$
\mathfrak{D}^{n}\left[x^{2}\right](0)=0, \quad n \geq 1 \text {. }
$$

Comparing (4.11) and (4.13) we can see the highly nonlinear behavior of the nested derivatives, since even the addition of 1 to $f(x)$ creates a completely different sequence of values, far more complex than the original.

We now start testing our result on some classical functions.

EXAMPLE 4.5 (elliptic functions). Let $f(x)=\sqrt{1-p^{2} \sin ^{2}(x)}, 0 \leq p \leq 1, a=$ 0 . We have, $f(0)=1$ and

$$
\begin{gathered}
h(\phi)=\int_{0}^{\phi} \frac{d \theta}{\sqrt{1-p^{2} \sin ^{2}(\theta)}}=F(p ; \phi), \\
H(p ; x)=\operatorname{am}(p ; x),
\end{gathered}
$$

where $F(p ; \phi)$ is the incomplete elliptic integral of the first kind, and am $(p ; x)$ is the elliptic amplitude [29]

$$
\begin{aligned}
\operatorname{am}(p ; x) & =\arcsin [\operatorname{sn}(p ; x)]=\arccos [c n(p ; x)] \\
& =\arcsin \left[\frac{\sqrt{1-d n^{2}(p ; x)}}{p}\right]
\end{aligned}
$$

with $\operatorname{sn}(p ; x), c n(p ; x)$, and $d n(p ; x)$ denoting the Jacobian elliptic functions.

Computing $\mathbb{D}^{n}[f](0)$ with (2.6) gives

$$
\begin{aligned}
\mathbb{L}^{2 k+1}[f](0) & =0, \quad k \geq 0, \\
\mathbb{D}^{2 k}[f](0) & =(-1)^{k} p^{2} Q_{k}(p), \quad k \geq 1,
\end{aligned}
$$


where $Q_{k}(p)$ is a polynomial of degree 2(k-1) of the form

$$
Q_{k}(p)=p^{2(k-1)}+\cdots+2^{2(k-1)} .
$$

The first few $Q_{k}(p)$ are

$$
\begin{aligned}
& Q_{1}(p)=1, \\
& Q_{2}(p)=p^{2}+4, \\
& Q_{3}(p)=p^{4}+44 p^{2}+16, \\
& Q_{4}(p)=p^{6}+408 p^{4}+912 p^{2}+64, \\
& Q_{5}(p)=p^{8}+3688 p^{6}+307682 p^{4}+15808 p^{2}+256,
\end{aligned}
$$

and (4.1) implies that

$$
\operatorname{am}(p ; x)=z-p^{2} \frac{z^{3}}{3 !}+p^{2}\left(p^{2}+4\right) \frac{z^{5}}{5 !}-p^{2}\left(p^{4}+44 p^{2}+16\right) \frac{z^{7}}{7 !}+\cdots
$$

in agreement with the known expansions for $\operatorname{am}(p ; x)[11]$.

EXAMPLE 4.6 (the Lambert- $W$ function). Let $f(x)=e^{-x}(x+1)^{-1}, a=0$, $f(a)=1$. Here,

$$
h(x)=x e^{x}, \quad H(x)=L W(x),
$$

where by $L W(x)$ we denote the Lambert- $W$ function $[9,10,22]$. In this case, (2.6) gives

$$
\begin{aligned}
\mathbb{D}^{1}[f](0) & =-2, \\
\mathbb{D}^{2}[f](0) & =9, \\
\mathbb{D}^{3}[f](0) & =-64, \\
& \vdots \\
\mathbb{D}^{n}[f](0) & =[-(n+1)]^{n} .
\end{aligned}
$$

From (4.1) we conclude that

$$
L W(z)=\sum_{n \geq 1}(-1)^{n-1} n^{n-1} \frac{z^{n}}{n !}
$$

EXAMPLE 4.7. We now derive a well-known result [1] about reversion of series. If we take

$$
h(x)=a_{1} x+a_{2} x^{2}+a_{3} x^{3}+a_{4} x^{4}+a_{5} x^{5}+a_{6} x^{6}+a_{7} x^{7}+\cdots,
$$


where $a_{1} \neq 0$, then

$$
f(x)=\frac{1}{h^{\prime}(x)}=\frac{1}{a_{1}+2 a_{2} x+3 a_{3} x^{2}+4 a_{4} x^{3}+5 a_{5} x^{4}+6 a_{6} x^{5}+7 a_{7} x^{6}+\cdots},
$$

$a=0, f(0)=1 / a_{1}$, and from (2.6), we get

$$
\begin{aligned}
\mathbb{D}^{1}[f](0) & =-2 \frac{a_{2}}{\left(a_{1}\right)^{2}}, \\
\mathfrak{D}^{2}[f](0) & =6 \frac{2\left(a_{2}\right)^{2}-a_{1} a_{3}}{\left(a_{1}\right)^{4}}, \\
\mathbb{D}^{3}[f](0) & =24 \frac{5 a_{1} a_{2} a_{3}-\left(a_{1}\right)^{2} a_{4}-5\left(a_{2}\right)^{3}}{\left(a_{1}\right)^{6}}, \\
\mathbb{D}^{4}[f](0) & =120 \frac{6\left(a_{1}\right)^{2} a_{2} a_{3}+3\left(a_{1} a_{3}\right)^{2}+14\left(a_{2}\right)^{4}-\left(a_{1}\right)^{3} a_{5}-21 a_{1}\left(a_{2}\right)^{2} a_{3}}{\left(a_{1}\right)^{8}} .
\end{aligned}
$$

Hence,

$$
\begin{aligned}
H(z)= & \frac{1}{a_{1}} z-\frac{a_{2}}{\left(a_{1}\right)^{3}} z^{2}+\frac{2\left(a_{2}\right)^{2}-a_{1} a_{3}}{\left(a_{1}\right)^{5}} z^{3} \\
& +\frac{5 a_{1} a_{2} a_{3}-\left(a_{1}\right)^{2} a_{4}-5\left(a_{2}\right)^{3}}{\left(a_{1}\right)^{7}} z^{4} \\
& +\frac{6\left(a_{1}\right)^{2} a_{2} a_{3}+3\left(a_{1} a_{3}\right)^{2}+14\left(a_{2}\right)^{4}-\left(a_{1}\right)^{3} a_{5}-21 a_{1}\left(a_{2}\right)^{2} a_{3}}{\left(a_{1}\right)^{9}} z^{5}+\cdots
\end{aligned}
$$

REMARK 4.8. An explicit formula for the $n$th term is given by Morse and Feshbach in [24, Part 1, pages 411-413],

$$
\begin{aligned}
b_{n}=\frac{1}{n\left(a_{1}\right)^{n}} \sum_{s, t, u, \ldots} & (-1)^{s+t+u+\cdots} \frac{n(n+1) \cdots(n-1+s+t+u+\cdots)}{s ! t ! u ! \cdots} \\
\times & \left(\frac{a_{2}}{a_{1}}\right)^{s}\left(\frac{a_{3}}{a_{1}}\right)^{t} \cdots, \\
& s+2 t+3 u+\cdots=n-1 .
\end{aligned}
$$

EXAMPLE 4.9 (the error function, $\operatorname{erf}(x)$ ). We now have

$$
\begin{gathered}
h(x)=\operatorname{erf}(x)=\frac{2}{\sqrt{\pi}} \int_{0}^{x} e^{-t^{2}} d t \\
f(x)=\frac{\sqrt{\pi}}{2} e^{x^{2}}, \quad a=0, \quad f(a)=\frac{\sqrt{\pi}}{2},
\end{gathered}
$$


and (2.6) gives,

$$
\mathbb{D}^{n}[f](0)= \begin{cases}0, & n=2 k+1, k \geq 0, \\ \left(\frac{\sqrt{\pi}}{2}\right)^{n} A_{k}, & n=2 k, k \geq 0,\end{cases}
$$

where

$$
\begin{aligned}
& A_{0}=1, \quad A_{1}=2, \quad A_{2}=28, \quad A_{3}=1016, \quad A_{4}=69904, \\
& A_{5}=7796768, \quad A_{6}=1282366912, \quad A_{7}=291885678464 .
\end{aligned}
$$

From (4.1), we get

$$
H(z)=\sum_{n \geq 0} A_{n}\left(\frac{\sqrt{\pi}}{2}\right)^{2 n+1} \frac{z^{2 n+1}}{(2 n+1) !} .
$$

Our result agrees with other authors calculations [3, 7, 13, 15, 30].

We will now extend (4.1) to a more general result.

COROLlARY 4.10. Let $h(x)=\int_{a}^{x}(1 / f(t)) d t, z_{0}=h(b)$, with $f(b) \neq 0$, $\pm \infty$, and its inverse $H(x)=h^{-1}(x)$. Then,

$$
H(z)=b+f(b) \sum_{n \geq 1} \mathbb{D}^{n-1}[f](b) \frac{\left(z-z_{0}\right)^{n}}{n !},
$$

where $\left|z-z_{0}\right|<\varepsilon$, for some $\varepsilon>0$.

Proof. Consider the function

$$
u(x)=h(x)-z_{0}
$$

which satisfies $u(b)=0$, and its inverse $U(x)=u^{-1}(x)$. Since $f(b) \neq 0, \pm \infty$, we can apply (4.1) to $u(x)$ and conclude that

$$
U(z)=b+f(b) \sum_{n \geq 1} \mathbb{D}^{n-1}[f](b) \frac{z^{n}}{n !} .
$$

All that is left is to see the relation between $U(z)$ and $H(z)$. Suppose that $u(x)=y$. Then

$$
y=u(x)=h(x)-z_{0}, \quad h(x)=y+z_{0}, \quad x=H\left(y+z_{0}\right),
$$

and therefore,

$$
U(y)=H\left(y+z_{0}\right)
$$

or

$$
H(z)=U\left(z-z_{0}\right)
$$

and (4.32) follows. 
We will now use our results to get some power series expansions that have not been studied before .

EXAMPLE 4.11 (the incomplete Gamma function, $\gamma(v ; x)$ ). We have

$$
\begin{gathered}
h(v ; x)=\gamma(v ; x) \equiv \int_{0}^{x} e^{-t} t^{v-1} d t, \quad v>0, x \geq 0, \\
f(v ; x)=e^{x} x^{1-v}, \quad a=0 .
\end{gathered}
$$

Since

$$
f(v ; a)= \begin{cases}0, & 0<v<1 \\ \infty, & v>1\end{cases}
$$

we cannot apply (4.1). Choosing $b=1, z_{0}(v)=\gamma(v ; 1), f(v ; b)=e$, we conclude from (4.32) that

$$
H(v ; z)=1+e \sum_{n \geq 1} \mathbb{D}^{n-1}[f](1) \frac{\left[z-z_{0}(v)\right]^{n}}{n !} .
$$

We use (2.6) to compute the first few $\mathbb{L}^{n}[f](1)$ and obtain

$$
\mathfrak{L}^{n}[f](1)=e^{n} Q_{n}(v),
$$

where $Q_{n}(v)$ is a polynomial of degree $n$,

$$
\begin{aligned}
& Q_{1}(v)=2-v, \\
& Q_{2}(v)=7-7 v+2 v^{2}, \\
& Q_{3}(v)=36-53 v+29 v^{2}-6 v^{3}, \\
& Q_{4}(v)=245-474 v+375 v^{2}-146 v^{3}+24 v^{4}, \\
& Q_{5}(v)=2076-4967 v+5104 v^{2}-2847 v^{3}+874 v^{4}-120 v^{5},
\end{aligned}
$$

and we can write

$$
H(v ; z)=1+\sum_{n \geq 1} e^{n} Q_{n-1}(v) \frac{\left[z-z_{0}(v)\right]^{n}}{n !} .
$$

EXAMPLE 4.12 (the sine integral, $\operatorname{Si}(x)$ ). In this case,

$$
\begin{aligned}
& h(x)=\operatorname{Si}(x)=\int_{0}^{x} \frac{\sin (t)}{t} d t, \\
& f(x)=\frac{x}{\sin (x)}, \quad a=0 .
\end{aligned}
$$

For this example, $f(a)$ is well defined, but to simplify the calculations we choose $b=\pi / 2, z_{0}=\operatorname{Si}(\pi / 2) \simeq 1.37076216$. Then,

$$
f(b)=\frac{\pi}{2}, \quad \mathbb{D}^{n}[f]\left(\frac{\pi}{2}\right)=Q_{n}(\pi),
$$


where $Q_{n}(x)$ is once again a polynomial,

$$
\begin{aligned}
& Q_{1}(x)=1, \\
& Q_{2}(x)=1+\frac{1}{4} x^{2}, \\
& Q_{3}(x)=1+\frac{7}{4} x^{2}, \\
& Q_{4}(x)=1+8 x^{2}+\frac{9}{16} x^{4}, \\
& Q_{5}(x)=1+\frac{61}{2} x^{2}+\frac{159}{16} x^{4}, \\
& Q_{6}(x)=1+\frac{423}{4} x^{2}+\frac{1671}{16} x^{4}+\frac{225}{64} x^{6},
\end{aligned}
$$

and from (4.32), we obtain

$$
H(z)=\frac{\pi}{2}+\frac{\pi}{2} \sum_{n \geq 1} Q_{n}(\pi) \frac{\left(z-z_{0}\right)^{n}}{n !} .
$$

EXAMPLE 4.13 (the logarithm integral, li $(x)$ ). From the definition,

$$
\begin{aligned}
& h(x)=\operatorname{li}(x) \equiv \int_{0}^{x} \frac{1}{\ln (t)} d t, \\
& f(x)=\ln (x), \quad a=0 .
\end{aligned}
$$

In this case, $f(a)=-\infty$, so we must choose $b$. A natural candidate is $b=e$, and then

$$
\begin{gathered}
f(b)=1, \quad z_{0}=\operatorname{li}(e) \simeq 1.895117816, \\
\mathbb{D}^{n}[f](e)=e^{-n} A_{n}
\end{gathered}
$$

with

$$
\begin{aligned}
& A_{1}=1, \quad A_{2}=0, \quad A_{3}=-1, \quad A_{4}=2, \quad A_{5}=1, \\
& A_{6}=-26, \quad A_{7}=99, \quad A_{8}=90, \quad A_{9}=-3627,
\end{aligned}
$$

and we have

$$
H(z)=e+\sum_{n \geq 1} A_{n} \frac{\left(z-z_{0}\right)^{n}}{n !} .
$$

EXAMPLE 4.14 (the incomplete Beta function, $B(\nu, \mu ; x)$ ). By definition,

$$
h(\nu, \mu ; x)=B(\nu, \mu ; x) \equiv \int_{0}^{x} t^{\nu-1}(1-t)^{\mu-1} d t, \quad 0 \leq x<1
$$

and hence

$$
f(\nu, \mu ; x)=x^{1-\nu}(1-x)^{1-\mu}, \quad a=0 .
$$


To avoid the possible singularities at $x=0$ and $x=1$, we consider $b=1 / 2$, and therefore,

$$
\begin{aligned}
f(\nu, \mu ; b) & =\frac{1}{4} 2^{v+\mu}, \\
z_{0}(\nu, \mu) & =B\left(\nu, \mu ; \frac{1}{2}\right) .
\end{aligned}
$$

The $\mathbb{D}$ algorithm now gives

$$
\mathbb{D}^{n}[f]\left(\frac{1}{2}\right)=2^{n(v+\mu-1)} Q_{n}(\nu, \mu)
$$

with $Q_{n}(\nu, \mu)$ a multivariate polynomial of degree $n$,

$$
\begin{aligned}
Q_{1}(\nu, \mu)= & \mu-v, \\
Q_{2}(\nu, \mu)= & -2+v-4 v \mu+\mu+2 \mu^{2}+2 v^{2}, \\
Q_{3}(\nu, \mu)= & (\mu-v)\left(6 \mu^{2}-12 v \mu+7 \mu-12+6 v^{2}+7 v\right), \\
Q_{4}(\nu, \mu)= & 16-46 v \mu^{2}-46 v^{2} \mu-63 \mu^{2}-22 \mu+154 v \mu \\
& -96 v \mu^{3}-96 v^{3} \mu+144 v^{2} \mu^{2}-22 v \\
& -63 v^{2}+24 v^{4}+46 v^{3}+24 \mu^{4}+46 \mu^{3}, \\
Q_{5}(\nu, \mu)= & (\mu-v)\left(120 \mu^{4}+326 \mu^{3}-480 v \mu^{3}+720 v^{2} \mu^{2}-323 \mu^{2}\right. \\
& \quad-326 v \mu^{2}-362 \mu-480 v^{3} \mu+1154 v \mu-326 v^{2} \mu \\
& \left.\quad-323 v^{2}+240+120 v^{4}+326 v^{3}-362 v\right)
\end{aligned}
$$

and we have

$$
H(z)=\frac{1}{2}+\frac{1}{4} 2^{r+\mu} \sum_{n \geq 1} 2^{n(r+\mu-1)} Q_{n}(r, \mu) \frac{\left(z-z_{0}\right) n}{n !} .
$$

5. Conclusion. We have presented a simple method for computing the series expansion for the inverses of functions and have given a Maple procedure to generate the coefficients in these expansions. We showed several examples of the method applied to elementary and special functions and stated the first few terms of the series in each case.

ACKNOWLEDGMENT. I wish to express my gratitude to the referees for their extremely valuable suggestions and comments on previous versions of this work.

\section{REFERENCES}

[1] M. Abramowitz and I. A. Stegun (eds.), Handbook of Mathematical Functions with Formulas, Graphs, and Mathematical Tables, Dover Publications, New York, 1992.

[2] G. E. Andrews, Identities in combinatorics. II. A q-analog of the Lagrange inversion theorem, Proc. Amer. Math. Soc. 53 (1975), no. 1, 240-245. 
[3] J. M. Blair, C. A. Edwards, and J. H. Johnson, Rational Chebyshev approximations for the inverse of the error function, Math. Comp. 30 (1976), no. 136, 827830.

[4] G. R. Blakley, Formal solution of nonlinear simultaneous equations: Reversion of series in several variables, Duke Math. J. 31 (1964), 347-357.

[5] R. P. Brent and H. T. Kung, $O\left((n \log n)^{3 / 2}\right)$ algorithms for composition and reversion of power series, Analytic Computational Complexity (Proc. Sympos., Carnegie-Mellon Univ., Pittsburgh, Pa, 1975), Academic Press, New York, 1976, pp. 217-225.

[6]___ Fast algorithms for manipulating formal power series, J. Assoc. Comput. Mach. 25 (1978), no. 4, 581-595.

[7] L. Carlitz, The inverse of the error function, Pacific J. Math. 13 (1963), 459-470.

[8] C. C.-A. Cheng, J. H. McKay, J. Towber, S. S.-S. Wang, and D. L. Wright, Reversion of power series and the extended Raney coefficients, Trans. Amer. Math. Soc. 349 (1997), no. 5, 1769-1782.

[9] R. M. Corless, G. H. Gonnet, D. E. G. Hare, D. J. Jeffrey, and D. E. Knuth, On the Lambert $W$ function, Adv. Comput. Math. 5 (1996), no. 4, 329-359.

[10] R. M. Corless, D. J. Jeffrey, and D. E. Knuth, A sequence of series for the Lambert $W$ function, Proceedings of the 1997 International Symposium on Symbolic and Algebraic Computation (Kihei, Hawaii), ACM Press, New York, 1997, pp. 197-204.

[11] H. T. Davis, Introduction to Nonlinear Differential and Integral Equations, Dover Publications, New York, 1962.

[12] J. W. Dettman, Applied Complex Variables, Dover Publications, New York, 1984.

[13] D. Dominici, The inverse of the cumulative standard normal probability function, Integral Transform. Spec. Funct. 14 (2003), no. 4, 281-292.

[14] R. H. Estes and E. R. Lancaster, Some generalized power series inversions, SIAM J. Numer. Anal. 9 (1972), 241-247.

[15] H. E. Fettis, A stable algorithm for computing the inverse error function in the "tail-end" region, Math. Comp. 28 (1974), 585-587.

[16] D. C. Fielder, Tabulation of coefficients for operations on Taylor series, Math. Comp. 14 (1960), 339-345.

[17] A. R. Forsyth, Theory of Functions of a Complex Variable. Vols. 1 and 2, 3rd ed., Dover Publications, New York, 1965.

[18] I. Gessel, A noncommutative generalization and q-analog of the Lagrange inversion formula, Trans. Amer. Math. Soc. 257 (1980), no. 2, 455-482.

[19] I. Gessel and D. Stanton, Applications of q-Lagrange inversion to basic hypergeometric series, Trans. Amer. Math. Soc. 277 (1983), no. 1, 173-201.

[20] _ Another family of q-Lagrange inversion formulas, Rocky Mountain J. Math. 16 (1986), no. 2, 373-384.

[21] I. M. Gessel, A combinatorial proof of the multivariable Lagrange inversion formula, J. Combin. Theory Ser. A 45 (1987), no. 2, 178-195.

[22] D. J. Jeffrey, D. E. G. Hare, and R. M. Corless, Unwinding the branches of the Lambert $W$ function, Math. Sci. 21 (1996), no. 1, 1-7.

[23] Ch. Krattenthaler, Operator methods and Lagrange inversion: a unified approach to Lagrange formulas, Trans. Amer. Math. Soc. 305 (1988), no. 2, 431-465.

[24] P. M. Morse and H. Feshbach, Methods of Theoretical Physics. 2 volumes, McGrawHill, New York, 1953.

[25] R. M. Redheffer, Reversion of power series, Amer. Math. Monthly 69 (1962), no. 5, 423-425. 
[26] J. B. Reynolds, Reversion of series with applications, Amer. Math. Monthly 51 (1944), no. 10, 578-580.

[27] B. Salvy, Fast computation of some asymptotic functional inverses, J. Symbolic Comput. 17 (1994), no. 3, 227-236.

[28] B. Salvy and J. Shackell, Symbolic asymptotics: multiseries of inverse functions, J. Symbolic Comput. 27 (1999), no. 6, 543-563.

[29] J. Spanier and K. B. Oldham, An Atlas of Functions, Hemisphere Publishing, New York, 1987.

[30] A. Strecok, On the calculation of the inverse of the error function, Math. Comp. 22 (1968), 144-158.

[31] N. M. Temme, Asymptotic inversion of incomplete gamma functions, Math. Comp. 58 (1992), no. 198, 755-764.

[32] _ Asymptotic inversion of the incomplete beta function, J. Comput. Appl. Math. 41 (1992), no. 1-2, 145-157.

[33] C. E. Van Orstrand, Reversion of power series, Philos. Mag. 19 (1910), no. 6, 366376.

[34] E. T. Whittaker, On the reversion of series, Gaz. Mat. 12 (1951), no. 50, 1-1.

[35] D. Wright, The tree formulas for reversion of power series, J. Pure Appl. Algebra 57 (1989), no. 2, 191-211.

Diego Dominici: Department of Mathematics, State University of New York at New Paltz, New Paltz, NY 12561-2443, USA

E-mail address: dominicd@newpa 7 tz.edu 


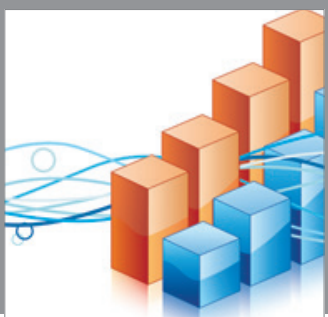

Advances in

Operations Research

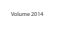

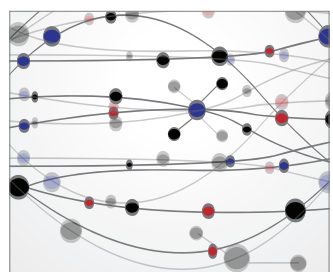

\section{The Scientific} World Journal
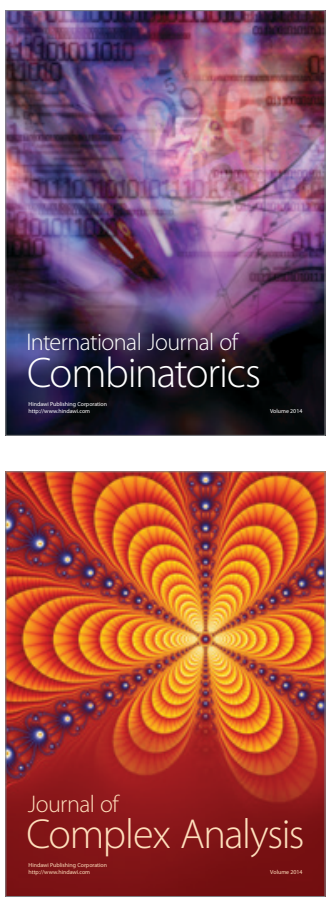

International Journal of

Mathematics and

Mathematical

Sciences
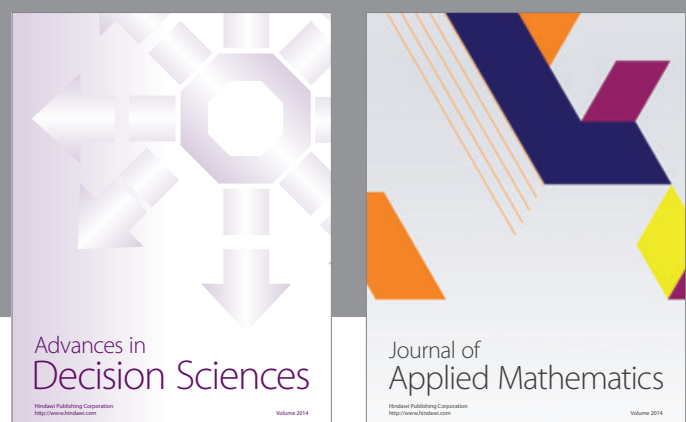

Journal of

Applied Mathematics
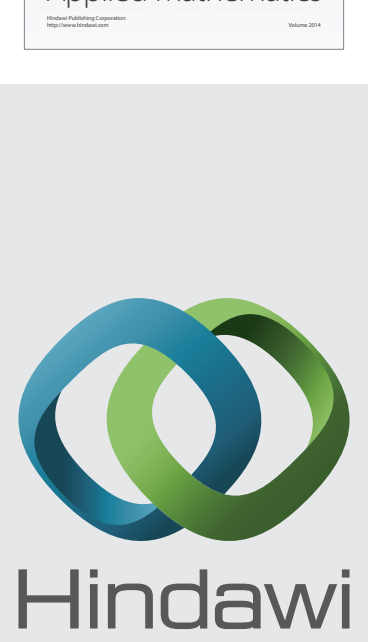

Submit your manuscripts at http://www.hindawi.com
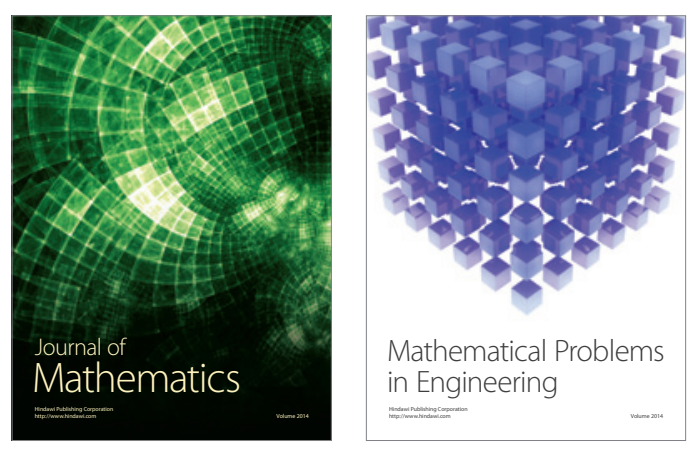

Mathematical Problems in Engineering
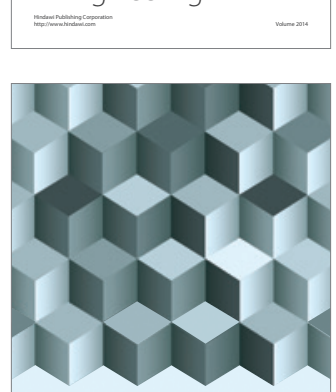

Journal of

Function Spaces
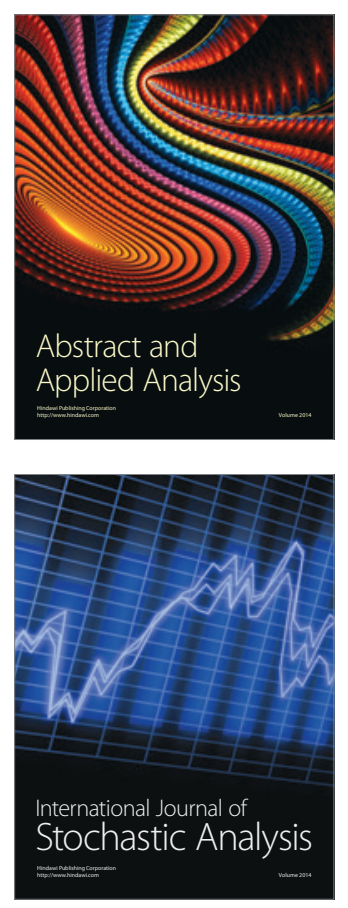

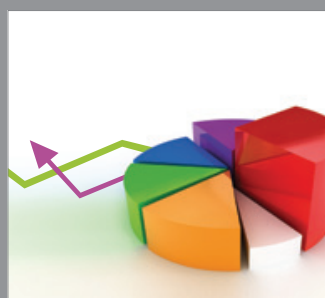

ournal of

Probability and Statistics

Promensencen
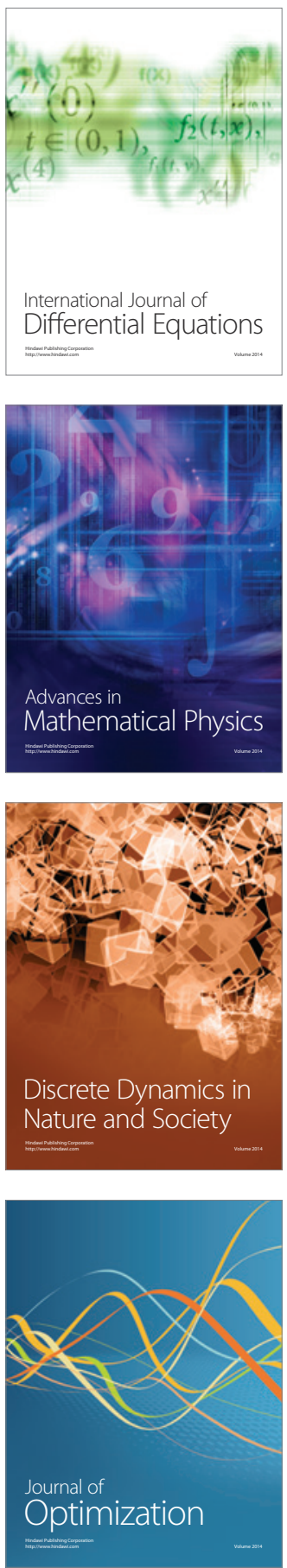